\title{
Kondo Resonance Decoherence by an External Potential
}

\author{
R. C. Monreal and F. Flores \\ Departamento de Física Teórica de la Materia Condensada, C-V . \\ Universidad Autónoma de Madrid, E-28049 Madrid, Spain.
}

September 16, 2018

\begin{abstract}
The Kondo problem, for a quantum dot (QD), subjected to an external bias, is analyzed in the limit of infinite Coulomb repulsion by using a consistent equations of motion method based on a slave-boson Hamiltonian. Utilizing a strict perturbative solution in the leads-dot coupling, $T$, up to $T^{4}$ and $T^{6}$ orders, we calculate the QD spectral density and conductance, as well as the decoherent rate that drive the system from the strong to the weak coupling regime. Our results indicate that the weak coupling regime is reached for voltages larger than a few units of the Kondo temperature.
\end{abstract}

PACS: 72.15.Qm, 73.23.Hk, 75.20.Hr 


\section{Introduction}

The transport of electrons through a quantum dot (QD) is a subject of increasing interest for its many applications in nanotechnology. Although there exists exact solutions for the ground state of the QD connected to metallic leads [1, no such methods are applicable to nonequilibrium systems. Typical cases are those of (a) a bias or (b) an electromagnetic radiation between the leads of a quantum well [2. It has been suggested that, in case (a), two Kondo resonances appear, each one associated with every lead [3], 4]. Some experimental evidence has been presented supporting this view [5]. Still, it is not well understood how inelastic (decoherent) processes modify the strongcoupling regime associated with the Kondo resonance. To our knowledge, there are only a few theoretical approaches to the problem. The non-crossing approximation (NCA) [3], [6], being essentially numerical, can give analytical results only in the limit $\operatorname{Ln}\left(V / T_{K}\right)>>1$ ( $\mathrm{V}$ being the applied bias and $T_{K}$ the Kondo energy), the same limit in which renormalized perturbation theory (RPT) [7], [8] is valid. It is important, however, to have analytical results for the decoherence rate, not only to make theoretical predictions, but also to be able to interpret correctly the results of calculations done for more complicated situations, i.e., when time- dependent potentials are applied to the QD [9]-[12]. A version of the nonequilibrium Kondo problem has been solved exactly to all orders in the applied bias $\mathrm{V}$ in [13].

In this article, we address the nonequilibrium Kondo problem for a QD in the limit of infinite Coulomb repulsion $\mathrm{U}$, using an equation of motion (EOM) method based on a slave-boson Hamiltonian. By means of a strict perturbative solution in the leads-dot coupling, $T$, to orders $T^{4}$ and $T^{6}$, we have been able to identify the decoherent processes that broaden the Kondolevel, as a function of the applied bias, and break the strong-coupling regime. The EOM method has been applied before to the Kondo problem [14] but it was mainly used with approximations valid only for high temperatures [3], 15], 16]. We show that this method, when applied consistently to high orders, can be conveniently used in the nonequilibrium case, describing well the inelastic processes contributing to the Kondo resonance decoherence. We first check the method in the equilibrium regime, comparing our results with exact results using the numerical renormalization group (NRG) and also with the NCA. When used in the nonequilibrium case, we find that, for an applied bias larger than $2 T_{K}$, the system develops two-peaked resonant structures at $\pm V / 2$; we show, however, that decoherent processes take the system from 
the strong-coupling regime, where the spin of the impurity is quenched by the lead electrons, to the weak-coupling regime, if $V / T_{K}$ is larger than a few units. We conclude that our method provides an accurate and powerful tool to analyze nonequilibrium systems and time-dependent problems, situations where very few theoretical approaches are valid.

\section{Theory}

Our starting point for a QD level with infinite- $U$, coupled to two leads, is the slave-boson Hamiltonian for spin up and down electrons [17]:

$$
\hat{H}=\sum_{k \sigma} \epsilon_{k \sigma} \hat{c}_{k \sigma}^{\dagger} \hat{c}_{k \sigma}+\sum_{\sigma} \epsilon_{0} \hat{d}_{\sigma}^{\dagger} \hat{d}_{\sigma}+\sum_{k \sigma} T_{k}\left(\hat{c}_{k \sigma}^{\dagger} \hat{\chi}_{\sigma}+\hat{\chi}_{\sigma}^{\dagger} \hat{c}_{k \sigma}\right)
$$

where the operators $\hat{\chi}_{\sigma}$ and $\hat{\chi}_{\sigma}^{\dagger}$ are introduced in terms of an auxiliary boson field, $\hat{b}$ and $\hat{b}^{\dagger}$, as $\hat{\chi}_{\sigma}=\hat{b}^{\dagger} \hat{d}_{\sigma}, \hat{\chi}_{\sigma}^{\dagger}=\hat{d}_{\sigma}^{\dagger} \hat{b}$ and $k=k_{L}, k_{R}$ for left and right leads. In eq.(11), the operator $\hat{c}_{k \sigma}^{\dagger}\left(\hat{c}_{k \sigma}\right)$ creates (anhilates) an electron of momentum $k$ and spin $\sigma$ in one of the leads, while $\hat{d}_{\sigma}^{\dagger}\left(\hat{d}_{\sigma}\right)$ creates (anhilates) an electron of spin $\sigma$ in the dot. The constraint relation

$$
\hat{b}^{\dagger} \hat{b}+\sum_{\sigma} \hat{d}_{\sigma}^{\dagger} \hat{d}_{\sigma}=1
$$

is imposed to guarantee that no more than one electron occupies the QD since we are in the infinite- $\mathrm{U}$ limit.

The physical (retarded) Green function for this problem is defined as 18, 19

$$
<<\hat{\chi}_{\sigma}(t) ; \hat{\chi}_{\sigma}^{\dagger}\left(t^{\prime}\right)>>=-i<\hat{\chi}_{\sigma}^{\dagger}\left(t^{\prime}\right) \hat{\chi}_{\sigma}(t)+\hat{\chi}_{\sigma}(t) \hat{\chi}_{\sigma}^{\dagger}\left(t^{\prime}\right)>\theta\left(t-t^{\prime}\right)
$$

and its Fourier transform is written as $<<\hat{\chi}_{\sigma} ; \hat{\chi}_{\sigma}^{\dagger}>>(\omega)$. The EOM for this

Green function reads (omitting the argument $\omega$ in all the Green functions):

$$
\begin{array}{r}
\left(w-\epsilon_{0}\right)<<\hat{\chi}_{\sigma} ; \hat{\chi}_{\sigma}^{\dagger}>>=1-<\hat{n}_{-\sigma}>+\sum_{k} T_{k}<<\left(1-\hat{n}_{-\sigma}\right) \hat{c}_{k \sigma} ; \hat{\chi}_{\sigma}^{\dagger}>>+ \\
\sum_{k} T_{k}<<\hat{d}_{-\sigma}^{\dagger} \hat{d}_{\sigma} \hat{c}_{k-\sigma} ; \hat{\chi}_{\sigma}^{\dagger}>>
\end{array}
$$


where the new Green functions appearing on the right-hand side of eq.(44) are defined as in eq.(3). $\hat{n}_{-\sigma}$ is the number operator for electrons of spin $-\sigma$ in the QD and $\langle\hat{A}\rangle$ will denote the expectation value of $\hat{A}$ from now on. In a second step, the EOM for these Green functions lead to a second generation of Green functions, viz.,

$$
\begin{gathered}
\left(w-\epsilon_{k}\right)<<\hat{c}_{k \sigma} ; \hat{\chi}_{\sigma}^{\dagger}>>=T_{k}<<\hat{\chi}_{\sigma} ; \hat{\chi}_{\sigma}^{\dagger}>> \\
\left(w-\epsilon_{k}\right)<<\hat{d}_{-\sigma}^{\dagger} \hat{d}_{\sigma} \hat{c}_{k-\sigma} ; \hat{\chi}_{\sigma}^{\dagger}>>=-<\hat{\chi}_{-\sigma}^{\dagger} \hat{c}_{k-\sigma}>+ \\
\sum_{k^{\prime}} T_{k^{\prime}}<<\hat{\chi}_{\sigma} \hat{c}_{k^{\prime}-\sigma}^{\dagger} \hat{c}_{k-\sigma} ; \hat{\chi}_{\sigma}^{\dagger}>>+\sum_{k^{\prime}} T_{k^{\prime}}<<\hat{\chi}_{-\sigma}^{\dagger} \hat{c}_{k-\sigma} \hat{c}_{k^{\prime} \sigma} ; \hat{\chi}_{\sigma}^{\dagger}>> \\
\left(w-\epsilon_{k}\right)<<\hat{n}_{-\sigma} \hat{c}_{k \sigma} ; \hat{\chi}_{\sigma}^{\dagger}>>=\sum_{k^{\prime}} T_{k^{\prime}}<<\hat{\chi}_{-\sigma} \hat{c}_{k^{\prime}-\sigma}^{\dagger} \hat{c}_{k \sigma} ; \hat{\chi}_{\sigma}^{\dagger}>>+ \\
\sum_{k^{\prime}} T_{k^{\prime}}<<\hat{\chi}_{-\sigma}^{\dagger} \hat{c}_{k^{\prime}-\sigma} \hat{c}_{k \sigma} ; \hat{\chi}_{\sigma}^{\dagger}>>
\end{gathered}
$$

Note that the second generation of Green functions involve the excitation of two electrons in the leads. In our approximation to $O\left(T^{2}\right)$, we contract operators $\hat{c}_{k \sigma}, \hat{c}_{k^{\prime} \sigma}^{\dagger}$, where possible and obtain a closed equation for $<<\hat{\chi}_{\sigma} ; \hat{\chi}_{\sigma}^{\dagger}>>$. In particular we take $<<\hat{\chi}_{\sigma} \hat{c}_{k^{\prime}-\sigma}^{\dagger} \hat{c}_{k-\sigma} ; \hat{\chi}_{\sigma}^{\dagger}>>=\delta_{k, k^{\prime}}<$ $\hat{n}_{k-\sigma}><<\hat{\chi}_{\sigma} ; \hat{\chi}_{\sigma}^{\dagger}>>$ and $<<\hat{\chi}_{-\sigma}^{\dagger} \hat{c}_{k-\sigma} \hat{c}_{k^{\prime} \sigma} ; \hat{\chi}_{\sigma}^{\dagger}>>=<<\hat{\chi}_{-\sigma} \hat{c}_{k^{\prime}-\sigma}^{\dagger} \hat{c}_{k \sigma} ; \hat{\chi}_{\sigma}^{\dagger}>>=<<$ $\hat{\chi}_{-\sigma}^{\dagger} \hat{c}_{k^{\prime}-\sigma} \hat{c}_{k \sigma} ; \hat{\chi}_{\sigma}^{\dagger}>>=0$. This yields the following approximation to $<<$ $\hat{\chi}_{\sigma} ; \hat{\chi}_{\sigma}^{\dagger}>>$, which is exact to $O\left(T^{2}\right)$ (see Ref. [19]):

$$
<<\hat{\chi}_{\sigma} ; \hat{\chi}_{\sigma}^{\dagger}>>=\frac{1-<\hat{n}_{-\sigma}>-\sum_{k} \frac{T_{k}<\hat{\chi}_{-\sigma}^{\dagger} \hat{c}_{k-\sigma}>}{\omega-\epsilon_{k}}}{\omega-\epsilon_{0}-\Sigma_{0}-\sum_{k} \frac{T_{k}^{2}<\hat{n}_{k}>}{\omega-\epsilon_{k}}}
$$

where

$$
\Sigma_{0}=\sum_{k} \frac{T_{k}^{2}}{\omega-\epsilon_{k}}
$$

We can obtain $<<\hat{\chi}_{\sigma} ; \hat{\chi}_{\sigma}^{\dagger}>>$ to $O\left(T^{4}\right)$ by making all possible contractions of operators $\hat{c}_{k \sigma}, \hat{c}_{k^{\prime} \sigma}^{\dagger}$ in a third generation of Green functions. For instance, the EOM for $<<\hat{\chi}_{-\sigma}^{\dagger} \hat{c}_{k^{\prime}-\sigma} \hat{c}_{k \sigma} ; \hat{\chi}_{\sigma}^{\dagger}>>$ in eq.(17) is: 


$$
\begin{array}{r}
\left(\omega+\epsilon_{0}-\epsilon_{k}-\epsilon_{k^{\prime}}\right)<<\hat{\chi}_{-\sigma}^{\dagger} \hat{c}_{k^{\prime}-\sigma} \hat{c}_{k \sigma} ; \hat{\chi}_{\sigma}^{\dagger}>>=T_{k^{\prime}}<<\hat{n}_{-\sigma} \hat{c}_{k \sigma} ; \hat{\chi}_{\sigma}^{\dagger}>>- \\
T_{k^{\prime}}<<\hat{d}_{-\sigma}^{\dagger} \hat{d}_{\sigma} \hat{c}_{k^{\prime}-\sigma} ; \hat{\chi}_{\sigma}^{\dagger}>>+\sum_{k^{\prime \prime}} T_{k^{\prime \prime}}<<\hat{d}_{-\sigma}^{\dagger} \hat{d}_{\sigma} \hat{c}_{k^{\prime}-\sigma} \hat{c}_{k^{\prime \prime} \sigma}^{\dagger} \hat{c}_{k \sigma} ; \hat{\chi}_{\sigma}^{\dagger}>>- \\
\sum_{k^{\prime \prime}} T_{k^{\prime \prime}}<<\left(1-\hat{n}_{\sigma}\right) \hat{c}_{k^{\prime \prime}-\sigma}^{\dagger} \hat{c}_{k^{\prime}-\sigma} \hat{c}_{k \sigma} ; \hat{\chi}_{\sigma}^{\dagger}>>
\end{array}
$$

which is approximated by

$$
\begin{array}{r}
\left(\omega+\epsilon_{0}-\epsilon_{k}-\epsilon_{k^{\prime}}\right)<<\hat{\chi}_{-\sigma}^{\dagger} \hat{c}_{k^{\prime}-\sigma} \hat{c}_{k \sigma} ; \hat{\chi}_{\sigma}^{\dagger}>>=T_{k^{\prime}}<<\hat{n}_{-\sigma} \hat{c}_{k \sigma} ; \hat{\chi}_{\sigma}^{\dagger}>>- \\
T_{k^{\prime}}<<\hat{d}_{-\sigma}^{\dagger} \hat{d}_{\sigma} \hat{c}_{k^{\prime}-\sigma} ; \hat{\chi}_{\sigma}^{\dagger}>>+T_{k}<\hat{n}_{k}><<\hat{d}_{-\sigma}^{\dagger} \hat{d}_{\sigma} \hat{c}_{k^{\prime}-\sigma} ; \hat{\chi}_{\sigma}^{\dagger}>>- \\
T_{k^{\prime}}<\hat{n}_{k^{\prime}}><<\left(1-\hat{n}_{\sigma}\right) \hat{c}_{k \sigma} ; \hat{\chi}_{\sigma}^{\dagger}>>
\end{array}
$$

This procedure yields the Green functions already obtained in eqs.(5) (7) and a new one, $<<\hat{n}_{\sigma} \hat{c}_{k \sigma} ; \hat{\chi}_{\sigma}^{\dagger}>>$, which has to be calculated as in eq.(17). The first generation of Green functions are thus obtained as:

$$
\begin{gathered}
\left(\tilde{\omega}_{k}-\Sigma_{1}\left(\tilde{\omega}_{k}\right)\right)<<\hat{d}_{-\sigma}^{\dagger} \hat{d}_{\sigma} \hat{c}_{k-\sigma} ; \hat{\chi}_{\sigma}^{\dagger}>>=-<\hat{\chi}_{-\sigma}^{\dagger} \hat{c}_{k-\sigma}>+ \\
T_{k}<n_{k}><<\hat{\chi}_{\sigma} ; \hat{\chi}_{\sigma}^{\dagger}>>-T_{k}<\hat{n}_{k}>\sum_{k^{\prime}} \frac{T_{k^{\prime}}<<\hat{n}_{\sigma} \hat{c}_{k^{\prime} \sigma} ; \hat{\chi}_{\sigma}^{\dagger}>>}{\tilde{\omega}_{k}-\epsilon_{k^{\prime}}+\epsilon_{0}} \\
\left(\tilde{\omega}_{k}-\Sigma_{2}\left(\tilde{\omega}_{k}\right)\right)<<\hat{n}_{-\sigma} \hat{c}_{k \sigma} ; \hat{\chi}_{\sigma}^{\dagger}>>=\Sigma_{3}\left(\tilde{\omega}_{k}\right)<<\hat{n}_{\sigma} \hat{c}_{k \sigma} ; \hat{\chi}_{\sigma}^{\dagger}>>+ \\
T_{k}<\hat{n}_{k}>\sum_{k^{\prime}} \frac{T_{k^{\prime}}<<\hat{d}_{-\sigma}^{\dagger} \hat{d}_{\sigma} \hat{c}_{k^{\prime}-\sigma} ; \hat{\chi}_{\sigma}^{\dagger}>>}{\tilde{\omega}_{k}-\epsilon_{k^{\prime}}+\epsilon_{0}} \\
\left(\tilde{\omega}_{k}-\Sigma_{2}\left(\tilde{\omega}_{k}\right)\right)<<\hat{n}_{\sigma} \hat{c}_{k \sigma} ; \hat{\chi}_{\sigma}^{\dagger}>>=-<\hat{\chi}_{\sigma}^{\dagger} \hat{c}_{k \sigma}>+T_{k}<\hat{n}_{k}><<\hat{\chi}_{\sigma} ; \hat{\chi}_{\sigma}^{\dagger}>>+ \\
\Sigma_{3}\left(\tilde{\omega}_{k}\right)<<\hat{n}_{-\sigma} \hat{c}_{k \sigma} ; \hat{\chi}_{\sigma}^{\dagger}>>-T_{k}<\hat{n}_{k}>\sum_{k^{\prime}} \frac{T_{k^{\prime}}<<\hat{n}_{-\sigma} \hat{c}_{k^{\prime} \sigma} ; \hat{\chi}_{\sigma}^{\dagger}>>}{\tilde{\omega}_{k}-\epsilon_{k^{\prime}}+\epsilon_{0}}
\end{gathered}
$$

with $\tilde{\omega}_{k}=\omega-\epsilon_{k}$ from now on. Eqs.(12,14) define a set of coupled integral equations in the energy levels of the leads, $\epsilon_{k}$, whose solution yields the Green functions to be introduced in eq.(41). In these equations we have neglected expectation values of operators such as $<\hat{\chi}_{\sigma}^{\dagger} \hat{\chi}_{-\sigma} \hat{c}_{k-\sigma}^{\dagger} \hat{c}_{k \sigma}>$, which 
give small contributions to $<<\hat{\chi}_{\sigma} ; \hat{\chi}_{\sigma}^{\dagger}>>$ to $O\left(T^{4}\right)$ and have defined several self-energies given by:

$$
\Sigma_{i}\left(\tilde{\omega}_{k}\right)=\sum_{k^{\prime}} T_{k^{\prime}}^{2} \nu_{i}\left[\frac{1}{\tilde{\omega}_{k}-\epsilon_{k^{\prime}}+\epsilon_{0}}+\frac{1}{\tilde{\omega}_{k}+\epsilon_{k^{\prime}}-\epsilon_{0}}\right]
$$

where $\nu_{1}=1-<\hat{n}_{k^{\prime}}>, \nu_{2}=1$ and $\nu_{3}=<\hat{n}_{k^{\prime}}>,\left(\Sigma_{3}\left(\tilde{\omega}_{k}\right)=\Sigma_{2}\left(\tilde{\omega}_{k}\right)-\right.$ $\left.\Sigma_{1}\left(\tilde{\omega}_{k}\right)\right)$. These self-energies are associated with different Green functions and play a crutial role in the Kondo-resonance decoherence, as we will see below. Eqs. (12]14) reproduce the conventional $T^{2}$-approximation of [19 by neglecting all the terms proportional to $T^{3}$. One can get, however, a new (and effective) $T^{2}$-order near the Kondo resonance by neglecting the terms that couple these equations in an integral way. This approximation yields the following Green function:

$$
\begin{aligned}
& <<\hat{\chi}_{\sigma} ; \hat{\chi}_{\sigma}^{\dagger}>>= \\
& {\left[1-<\hat{n}_{-\sigma}>-\sum_{k} \frac{T_{k}<\hat{\chi}_{-\sigma}^{\dagger} \hat{c}_{k-\sigma}>}{\tilde{\omega}_{k}-\Sigma_{1}\left(\tilde{\omega}_{k}\right)}+\sum_{k} T_{k} \frac{\Sigma_{3}\left(\tilde{\omega}_{k}\right)<\hat{\chi}_{\sigma}^{\dagger} \hat{c}_{k \sigma}>}{\left(\tilde{\omega}_{k}-\Sigma_{2}\left(\tilde{\omega}_{k}\right)\right)\left(\tilde{\omega}_{k}-\Sigma_{4}\left(\tilde{\omega}_{k}\right)\right)}\right]} \\
& {\left[\omega-\epsilon_{0}-\Sigma_{0}-\sum_{k} \frac{T_{k}^{2}<\hat{n}_{k}>}{\tilde{\omega}_{k}-\Sigma_{1}\left(\tilde{\omega}_{k}\right)}+\sum_{k} \frac{T_{k}^{2}<\hat{n}_{k}>\Sigma_{3}\left(\tilde{\omega}_{k}\right)}{\left(\tilde{\omega}_{k}-\Sigma_{2}\left(\tilde{\omega}_{k}\right)\right)\left(\tilde{\omega}_{k}-\Sigma_{4}\left(\tilde{\omega}_{k}\right)\right)}\right]^{-1}(16)}
\end{aligned}
$$

with

$$
\Sigma_{4}\left(\tilde{\omega}_{k}\right)=\Sigma_{2}\left(\tilde{\omega}_{k}\right)+\frac{\Sigma_{3}\left(\tilde{\omega}_{k}\right)^{2}}{\tilde{\omega}_{k}-\Sigma_{2}\left(\tilde{\omega}_{k}\right)} .
$$

In eq.(16),$<n_{-\sigma}>$ and $<\hat{\chi}_{-\sigma}^{\dagger} \hat{c}_{k-\sigma}>$ have to be calculated self-consistently [14, 19]. At zero temperature and in a wide-band approximation, they are given by:

$$
<\hat{n}_{-\sigma}>=\int_{-\infty}^{0}-\frac{d \omega}{\pi} \operatorname{Im}<<\hat{\chi}_{\sigma} ; \hat{\chi}_{\sigma}^{\dagger}>>(\omega)
$$

and

$$
<\hat{\chi}_{-\sigma}^{\dagger} \hat{c}_{k-\sigma}>=\int_{-\infty}^{0}-\frac{d \omega}{\pi} \operatorname{Im} \frac{T_{k}<<\hat{\chi}_{\sigma} ; \hat{\chi}_{\sigma}^{\dagger}>>(\omega)}{\tilde{\omega}_{k}}
$$

where $\omega=0$ is the Fermi level. 


\section{Results}

In this section we first discuss some consequences that our approximation bears in the description of the Kondo peak. Notice that the $T^{2}$-order of eq. (8) is given by eq.(16) neglecting the last terms of the numerator and denominator and taking $\Sigma_{1}\left(\tilde{\omega}_{k}\right)=0$. These new terms yield, however, contributions that are proportional to $T^{2}$ around the Kondo peak. This can be seen by considering the self-energies $\Sigma_{1}, \Sigma_{2}$ and $\Sigma_{4}$, whose imaginary parts tend to broaden the Kondo-like peak associated with the factors $\sum_{k} \frac{T_{k}^{2}<\hat{n}_{k}>}{\tilde{\omega}_{k}-\Sigma_{i}\left(\tilde{\omega}_{k}\right)}$. In the $T^{4}$-order, eq. (15) yields, for $V=0, \epsilon_{0}<0,\left|\epsilon_{0}\right|>>\Delta(2 \Delta$ being the oneelectron level-width) and a wide-band approximation, $\Sigma_{2}=2 \Sigma_{0} \equiv-2 i \Delta$, $\Sigma_{3} \simeq \Sigma_{2}$ and $\Sigma_{1}=0$, if $\left|\omega-\epsilon_{k}\right|<<\left|\epsilon_{0}\right|$. Then, near the Fermi level, we obtain:

$$
\sum_{k} \frac{T_{k}^{2}<\hat{n}_{k}>}{\tilde{\omega}_{k}-\Sigma_{1}\left(\tilde{\omega}_{k}\right)} \simeq \frac{\Delta}{\pi} \operatorname{Ln}\left(\frac{D}{\omega}\right)
$$

$\mathrm{D}$ being the half-bandwidth, and

$$
\sum_{k} \frac{T_{k}^{2}<\hat{n}_{k}>}{\tilde{\omega}_{k}-\Sigma_{2}\left(\tilde{\omega}_{k}\right)} \frac{\Sigma_{3}\left(\tilde{\omega}_{k}\right)}{\tilde{\omega}_{k}-\Sigma_{4}\left(\tilde{\omega}_{k}\right)} \simeq-\frac{\Delta}{2 \pi} \operatorname{Ln}\left(\frac{\omega+4 i \Delta}{\omega}\right) .
$$

The important point is that the term given by eq.(21), which is neglected in the $T^{2}$-order approximation, also gives a $T^{2}$-contribution to $<<\hat{\chi}_{\sigma} ; \hat{\chi}_{\sigma}^{\dagger}>>$ going like $\operatorname{Ln}(\omega)$ at the Fermi energy. In particular, eq.(16) yields the Kondoenergy

$$
T_{K} \simeq D^{2 / 3}(4 \Delta)^{1 / 3} \exp \left[\frac{-2 \pi\left|\epsilon_{0}\right|}{3 \Delta}\right]
$$

intermediate between $\operatorname{Dexp}\left[-\pi\left|\epsilon_{0}\right| / \Delta\right]$ (the $T^{2}$-order) and the exact result of Haldane $(D \Delta)^{1 / 2} \exp \left[-\pi\left|\epsilon_{0}\right| / 2 \Delta\right][20]$.

A check of our solution, eq.(16), can be obtained by considering the case $V=0$; this equilibrium case can be compared with the NCA and with the numerically exact calculations of Ref. 21] using the NRG. Fig.1 shows our calculated spectral density for the Kondo regime defined by $\epsilon_{0}=-4 \Delta$, $\Delta=0.01$ and $D=1$ at zero temperature. Two cases, $T^{2}$-order and eq. (16) are shown as a function of $\omega / T_{K}$. Although for this particular case our value of $T_{K}$, eq.(22), is very similar to Haldane's, we use this last one from now on. Our results indicate that eq.(16) yields a much better spectral density for the Kondo-resonance than the $T^{2}$-order aproximation, with values very 
similar to the ones given by the NRG above the Fermi energy, although our results yield a too small value of the spectral density below the Fermi energy; this deviation extends to energies of around $2 T_{K}$ and suggests that a much more accurate solution is going to be obtained for voltages larger than this value (see below). Fig.2 shows the same results for a mixed-valence regime $\left(\epsilon_{0}=0, \Delta=0.01\right.$ and $\left.D=1\right)$ at zero temperature. In this case, eq.(16) yields an excellent approximation to the exact NRG-calculation and the Friedel-Langreth sum rule is satisfied with an accuracy better than $10 \%$. The agreement is even better in the empty orbital regime, Fig.3, where the sum rule is fulfilled to within $5 \%$ accuracy. We should mention that our present results are in much better agreement with the exact results than the ones obtained using Lacroix's approximation [14] and shown in [19].

Now we turn our attention to the nonequilibrium case $V \neq 0$ in the symmetric situation, where the chemical potentials of the left and right leads are $\pm V / 2$. A fully consistent solution of the case $V \neq 0$ requires the application of the Keldysh formalism, as explained in [19. The symmetric case can be analyzed, however, at the level of the above equations because, to good accuracy, $\left\langle n_{-\sigma}\right\rangle$ and $\left\langle\hat{\chi}_{-\sigma}^{\dagger} \hat{c}_{k-\sigma}\right\rangle$ do not vary with $\mathrm{V}$ for the values used here. We start by analyzing the self-energies $\Sigma_{1}$ and $\Sigma_{4}$ in the regime $\tilde{\omega}_{k} \rightarrow 0$, which is the relevant regime for analyzing the Kondo-resonance decoherence. It is easy to see that eq.(15) produces $\Sigma_{1}\left(\tilde{\omega}_{k} \rightarrow 0\right) \rightarrow 0$, as in the equilibrium case and therefore, we find no decoherence for $V \neq 0$. It is interesting to proceed further with the EOM method and see how these self-energies are modified. By going to $O\left(T^{6}\right)$, we find for $\Sigma_{1}$ and $\Sigma_{2}$ :

$$
\begin{gathered}
\Sigma_{1}\left(\tilde{\omega}_{k}\right)=\sum_{k^{\prime}} T_{k^{\prime}}^{2}\left[\frac{1-<\hat{n}_{k^{\prime}}>}{\tilde{\omega}_{k}-\epsilon_{k^{\prime}}+\epsilon_{-}\left(k^{\prime}\right)+i \Delta}+\frac{1-<\hat{n}_{k^{\prime}}>}{\tilde{\omega}_{k}+\epsilon_{k^{\prime}}-\epsilon_{+}\left(k^{\prime}\right)+i \Delta}\right]+ \\
2 \sum_{k^{\prime}, k^{\prime \prime}} \frac{T_{k^{\prime}}^{2}\left(1-<\hat{n}_{k^{\prime}}>\right)}{\tilde{\omega}_{k}-\epsilon_{k^{\prime}}+\epsilon_{-}\left(k^{\prime}\right)+i \Delta} \frac{1}{\tilde{\omega}_{k}+\epsilon_{k^{\prime \prime}}-\epsilon_{k^{\prime}}} \frac{T_{k^{\prime \prime}}^{2}\left(1-<\hat{n}_{k^{\prime \prime}}>\right)}{\tilde{\omega}_{k}+\epsilon_{k^{\prime \prime}}-\epsilon_{+}\left(k^{\prime \prime}\right)+i \Delta}+ \\
\sum_{k^{\prime}, k^{\prime \prime}, q} \frac{T_{k^{\prime}}^{2}\left(1-<\hat{n}_{k^{\prime}}>\right)}{\tilde{\omega}_{k}-\epsilon_{k^{\prime}}+\epsilon_{-}\left(k^{\prime}\right)+i \Delta} \frac{1}{\tilde{\omega}_{k}+\epsilon_{k^{\prime \prime}}-\epsilon_{k^{\prime}}} \frac{T_{k^{\prime \prime}}^{2}\left(1-<\hat{n}_{k^{\prime \prime}}>\right)}{\tilde{\omega}_{k}+\epsilon_{k^{\prime \prime}}-\epsilon_{+}\left(k^{\prime \prime}\right)+i \Delta} \times \\
\frac{1}{\tilde{\omega}_{k}+\epsilon_{k^{\prime \prime}}-\epsilon_{q}} \frac{T_{q}^{2}\left(1-<\hat{n}_{q}>\right)}{\tilde{\omega}_{k}-\epsilon_{q}+\epsilon_{-}(q)+i \Delta}+O\left(T^{8}\right)
\end{gathered}
$$

and

$$
\Sigma_{2}\left(\tilde{\omega}_{k}\right)=\sum_{k^{\prime}} T_{k^{\prime}}^{2}\left[\frac{1}{\tilde{\omega}_{k}-\epsilon_{k^{\prime}}+\epsilon_{-}\left(k^{\prime}\right)+i \Delta}+\frac{1}{\tilde{\omega}_{k}+\epsilon_{k^{\prime}}-\epsilon_{+}\left(k^{\prime}\right)+i \Delta}\right]+
$$


$2 \sum_{k^{\prime}, k^{\prime \prime}} \frac{T_{k^{\prime}}^{2}<\hat{\omega}_{k^{\prime}}>}{\tilde{\omega}_{k}-\epsilon_{k^{\prime}}+\epsilon_{-}\left(k^{\prime}\right)+i \Delta} \times \frac{1}{\tilde{\omega}_{k}+\epsilon_{k^{\prime \prime}}-\epsilon_{k^{\prime}}} \times \frac{T_{k^{\prime \prime}}^{2}<\hat{n}_{k^{\prime \prime}}>}{\tilde{\omega}_{k}+\epsilon_{k^{\prime \prime}}-\epsilon_{+}\left(k^{\prime \prime}\right)+i \Delta}+O\left(T^{8}\right)(24)$

where $\epsilon_{ \pm}\left(\epsilon_{k^{\prime}}\right)=\epsilon_{0}-\frac{\Delta}{\pi} \operatorname{Ln}\left(\frac{\epsilon_{k^{\prime}} \pm \tilde{\omega}_{k}}{D}\right)$. We also get $\Sigma_{3}\left(\tilde{\omega}_{k}\right)=\Sigma_{2}\left(\tilde{\omega}_{k}\right)-\Sigma_{1}\left(\tilde{\omega}_{k}\right)$, $\Sigma_{4}\left(\tilde{\omega}_{k}\right)=\Sigma_{2}\left(\tilde{\omega}_{k}\right)+\frac{\Sigma_{3}\left(\tilde{\omega}_{k}\right)^{2}}{\tilde{\omega}_{k}-\Sigma_{2}\left(\tilde{\omega}_{k}\right)}$, as before. These higher order self-energies give rise to the first non-zero contribution to the Kondo resonance decoherence, due to the cross terms between the right and left leads, as has already been pointed out in [3]. Eqs.(23) and (24) represents the first terms of an expansion for $\Sigma_{1}$ or $\Sigma_{2}$, using $\left(T_{k} / \epsilon_{0}\right)^{2}$ as the expansion parameter, and allows us to analyze the Kondo resonance decoherence as a function of an external bias. For $V=0$, we again find $\Sigma_{1}\left(\tilde{\omega}_{k} \rightarrow 0\right) \rightarrow 0$ and $\Sigma_{4}\left(\tilde{\omega}_{k} \rightarrow 0\right) \rightarrow 0$ but for $V \neq 0$ these self-energies develop non-negligible imaginary parts for $\tilde{\omega}_{k} \rightarrow 0$. In the bias regime, defined by $V<<\left|\tilde{\epsilon}_{0}\right|<\Delta$, we find

$$
\Sigma_{1}\left(\tilde{\omega}_{k} \rightarrow 0 ; V\right) \simeq-i\left(\frac{\Delta}{\tilde{\epsilon}_{0}}\right)^{2} \frac{V}{2 \pi}\left(1-2 \frac{\Delta}{\pi\left|\tilde{\epsilon}_{0}\right|} \operatorname{Ln} \frac{V}{\left|\tilde{\epsilon}_{0}\right|}\right) \equiv-i \gamma
$$

and $\Sigma_{2} \simeq-i 2 \Delta$. In eq.(25), $\tilde{\epsilon}_{0}$ is a renormalized level given approximately by $\tilde{\epsilon}_{0} \simeq \epsilon_{0}+\frac{\Delta}{\pi} \operatorname{Ln} \frac{\pi D}{\Delta}$. Fig.4 shows the evolution of the Kondo-like resonance spectral density for the same parameters used in Fig.1, but introducing different biases on the QD. Here we have used eq.(16) and the self-energies to $T^{6}$-order. Our results are compared with the NCA-calculations of Ref. [9], where available. The agreement between both approaches, for $\omega>0$, is remarkable. The difference between both cases can be attributed to the bandwidth used in [9], which is ten times smaller than ours. For $\omega<0$, however, the agreement is only reasonable at low bias (like in the equilibrium case shown in Fig.1), which improves as $V$ increases.

We have also calculated the QD-conductance which, in the symmetric configuration we are considering, is basically proportional to the mean value of the spectral density in the interval $(-V / 2, V / 2)$. Fig.5 shows the conductance $G$ in units of the conductance quantum $G_{0}=2 e^{2} / h$, as a function of $V / T_{K}$; a good fit is obtained by the expression:

$$
G / G_{0}=1 /\left(1+0.75 \operatorname{Ln}\left(1+\left(\frac{V}{2 T_{K}}\right)^{2}\right)\right),
$$

for large values of $V / T_{K}$. These values also show good agreement with the NCA-calculations of [7] for $V>2 T_{K}$, where our solution can be taken with great confidence, although in this case, $G / G_{0}$ behaves like $1 / L n^{2}\left(V / T_{K}\right)$ for very high voltages, $\operatorname{Ln}\left(V / T_{K}\right)>>1$. 
In addition, we have also addressed the important issue of strong versus weak coupling regimes, as a function of the bias. It has been argued [22] that the strong-coupling regime might subsist, even for high bias $(V>>$ $T_{K}$ ), if the decoherence rate $\gamma$ is smaller than $T^{*}=-V / 2+\sqrt{T_{K}^{2}+(V / 2)^{2}}$. With $\gamma$ given by eq. (25), the strong-coupling regime is obtained for $V \leq$ $\pi^{1 / 2} \frac{\left|\tilde{\epsilon}_{0}\right|}{\Delta} T_{K}$. This is a very stringent condition; for instance, for the values of the parameters in Figs.1a and 2, we find strong coupling for $V \leq 3.5 T_{K}$, for which the conductance is larger than $0.4 G_{0}$. We conclude that, when subjected to an external bias $V>T_{K}$, the QD-Kondo resonance develops a two-peaked structure at $\pm V / 2$ for $V>2 T_{K}$; the system, however, evolves quickly as a function of the voltage, moving from a strong-coupling regime with the spin impurity screened by the electrons of the leads, to a weakcoupling regime with that spin unscreened.

\section{Conclusions}

The EOM method provides an accurate approximation to the Kondo problem in and out of equilibrium situations, the only requirement being to include all the Green functions in a strict expansion in $T^{2}$. Our results to $T^{4}$-order in equilibrium provide a good description of the Kondo peak in the Kondo regime and an excellent aproximation to (numerically) exact results in the mixed-valence and empty-orbital regimes. In the nonequilibrium regime, it is necessary to carry the method to $O\left(T^{6}\right)$ to find the Kondo resonance decoherence, since it involves the coupling between the right and left electrodes through the QD. In particular, in our approach, the decoherence rate is found analytically to be nearly proportional to $V$, which indicates that the system is driven readily to the weak couping regime if $V \geq \pi^{1 / 2} \frac{\left|\tilde{\epsilon}_{0}\right|}{\Delta} T_{K}$, with the spin impurity unquenched by the electrons of the leads.

\section{Acknowledgements}

We are indebted to E.A. Anda and E.C. Goldberg for many fruitful discussions, to S. Davison for a critical reading of the manuscript and to T. Costi and P. Nordlander for providing us with their data. This work has been funded by the Spanish Comisión Interministerial de Ciencia y Tecnología under contracts MAT-2001-0665 and BFM-2001-0150. 


\section{References}

[1] A.C. Hewson in The Kondo Problem to Heavy Fermions (cambridge University Press, Cambridge, England, 1993).

[2] A. Kaminski, Yu.V. Nazarov and L.I. Glazman, Phys. Rev. Lett. 83, 384 (1999).

[3] Y. Meir, N. S. Wingreen and P.A. Lee, Phys. Rev. Lett. 17, 2601 (1993).

[4] Tatsuya Fujii and Kazuo Ueda, Phys. Rev. B 68, 155510 (2003).

[5] S. De Franceschi, R. Hanson, W.G. van der Wiel, J.M. Elzerman, J.J. Wipjkema, T. Fusijava, S. Tarucha and L.P. Kouwenhoven, Phys. Rev. Lett. 89, 156801 (2002).

[6] Ned. S. Wingreen and Yigal Meir, Phys. Rev. B 49, 11040 (1994).

[7] A. Rosch, J. Kroha and P. Wolfle, Phys. Rev. Lett. 87, 156802 (2001).

[8] J. Paaske, A. Rosch, J. Kroha and P. Wolfle, Phys. Rev. B 70, 155301 (2004).

[9] M. Plihal, D.C. Langreth and P. Nordlander, Phys. Rev. B 61, R13341 (2000).

[10] M. Plihal, D.C. Langreth and P. Nordlander, Phys. Rev. B 71, 165321 (2005).

[11] M.A. Cazalilla and J.B. Marston, Phys. Rev. Lett. 25, 256403 (2002).

[12] J. Merino and J.B. Marston, Phys. Rev. B 69, 115304 (2004).

[13] Avraham Shiller and Selman Hershfield, Phys. Rev. B 51,12898 (1995).

[14] C. Lacroix, J. Phys. F: Met. Phys. 11, 2389 (1981).

[15] Y. Meir, N.S. Wingreen and P.A. Lee, Phys. Rev. Lett. 66, 3048 (1991).

[16] R. Swirkowicz, J. Barnas and M. Wilczynski, Phys. Rev. B 68, 195318 (2003).

[17] P. Coleman, Phys. Rev. B 29, 3035 (1984). 
[18] N.E. Bickers, Rev. Mod. Phys. 59, 845 (1987).

[19] E.C. Goldberg, F. Flores and R. C. Monreal, Phys. Rev. B 71, 035112 (2005).

[20] F.D.M. Haldane, J. Phys. C 11, 5015 (1978).

[21] T.A. Costi, J. Kroha and P. Wolfle, Phys. Rev. B 53, 1850 (1996).

[22] P. Coleman, C. Hooley and O. Parcollet, Phys. Rev. Lett. 86, 4088 (2001). 


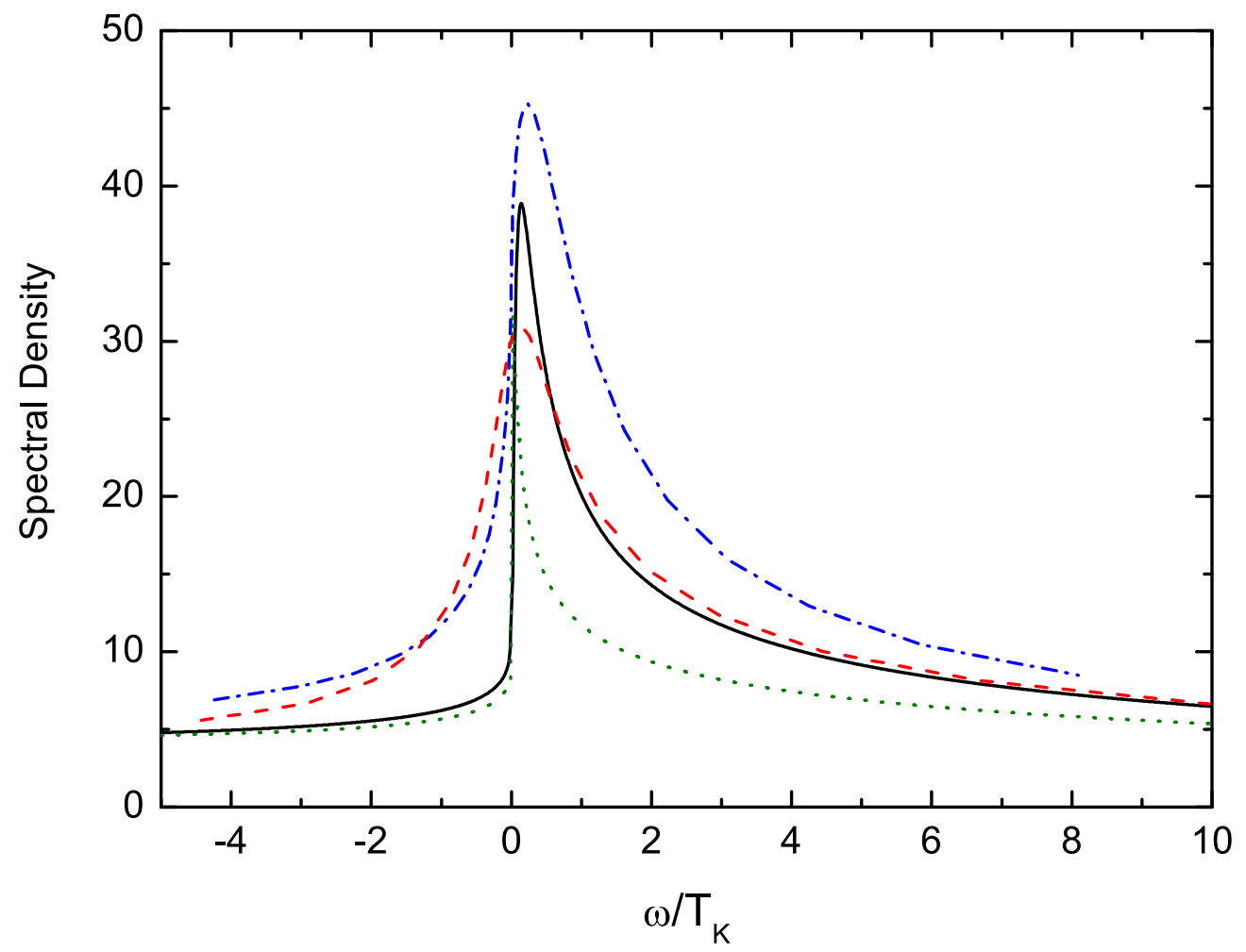

Figure 1: The spectral density in equilibrium as a function of $w / T_{K}$ for $D=1$ and $\Delta=0.01$ is plotted for the Kondo regime: $\epsilon_{0}=-4 \Delta, T_{K} / \Delta=$ $1.87 \times 10^{-2}$. Dotted line: $T^{2}$-EOM; full line: $T^{4}$-EOM; dashed line: NRG from [21]: dashed-dotted line: NCA from $[21]\left(T=10^{-6} D\right)$. 


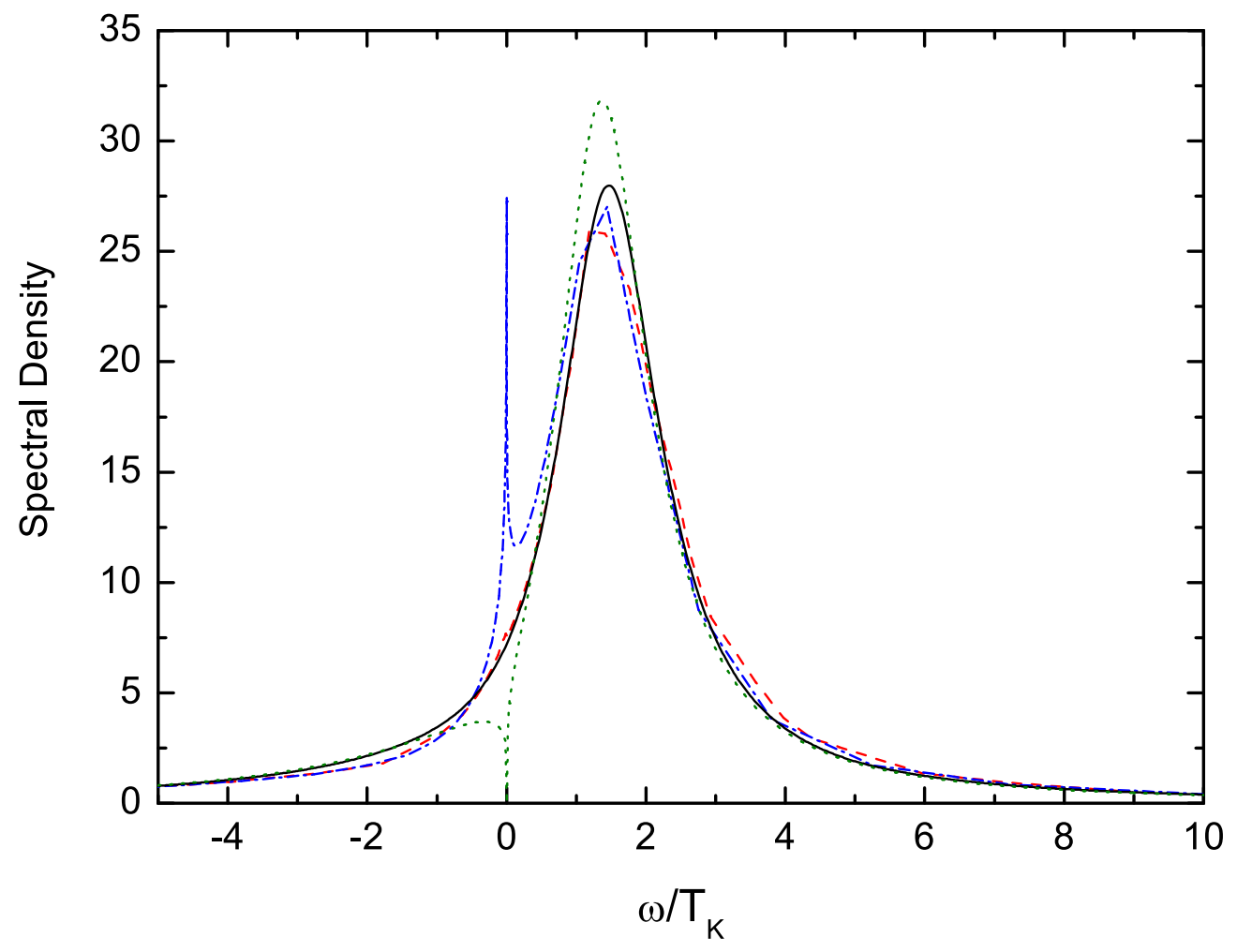

Figure 2: The spectral density in equilibrium as a function of $w / T_{K}$ for $D=1$ and $\Delta=0.01$ is plotted for the mixed-valence regime $\epsilon_{0}=0, T_{K} / \Delta=$ 1. Dotted line: $T^{2}$-EOM; full line: $T^{4}$-EOM; dashed line: NRG from [21]: dashed-dotted line: NCA from $[21]\left(T=10^{-6} D\right)$. 


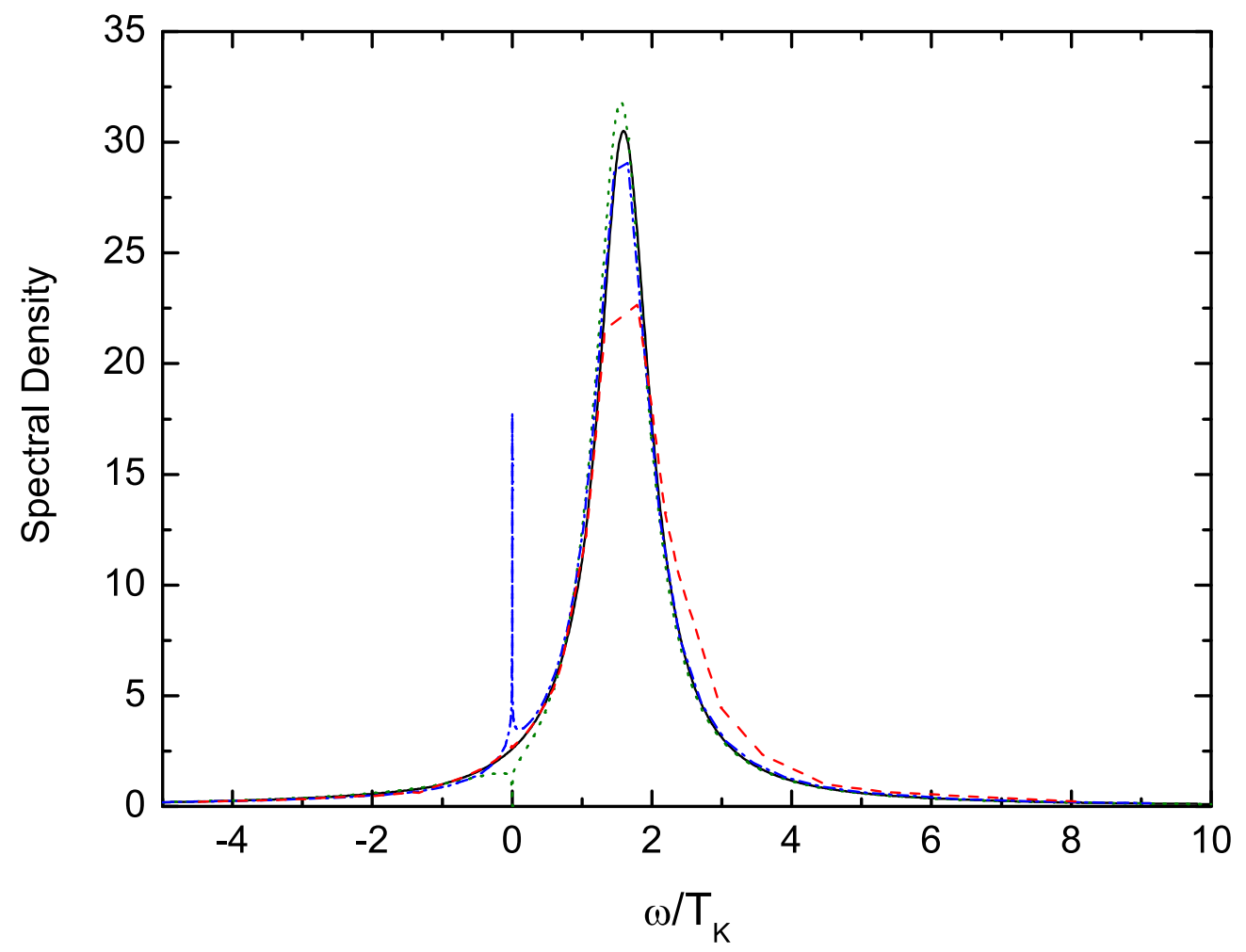

Figure 3: The spectral density in equilibrium as a function of $w / T_{K}$ for $D=1$ and $\Delta=0.01$ is plotted for the empty-orbital regime $\epsilon_{0}=2 \Delta$, $T_{K} / \epsilon_{0}=1$. Dotted line: $T^{2}$-EOM; full line: $T^{4}$-EOM; dashed line: NRG from [21]: dashed-dotted line: NCA from $[21]\left(T=10^{-6} D\right)$. 


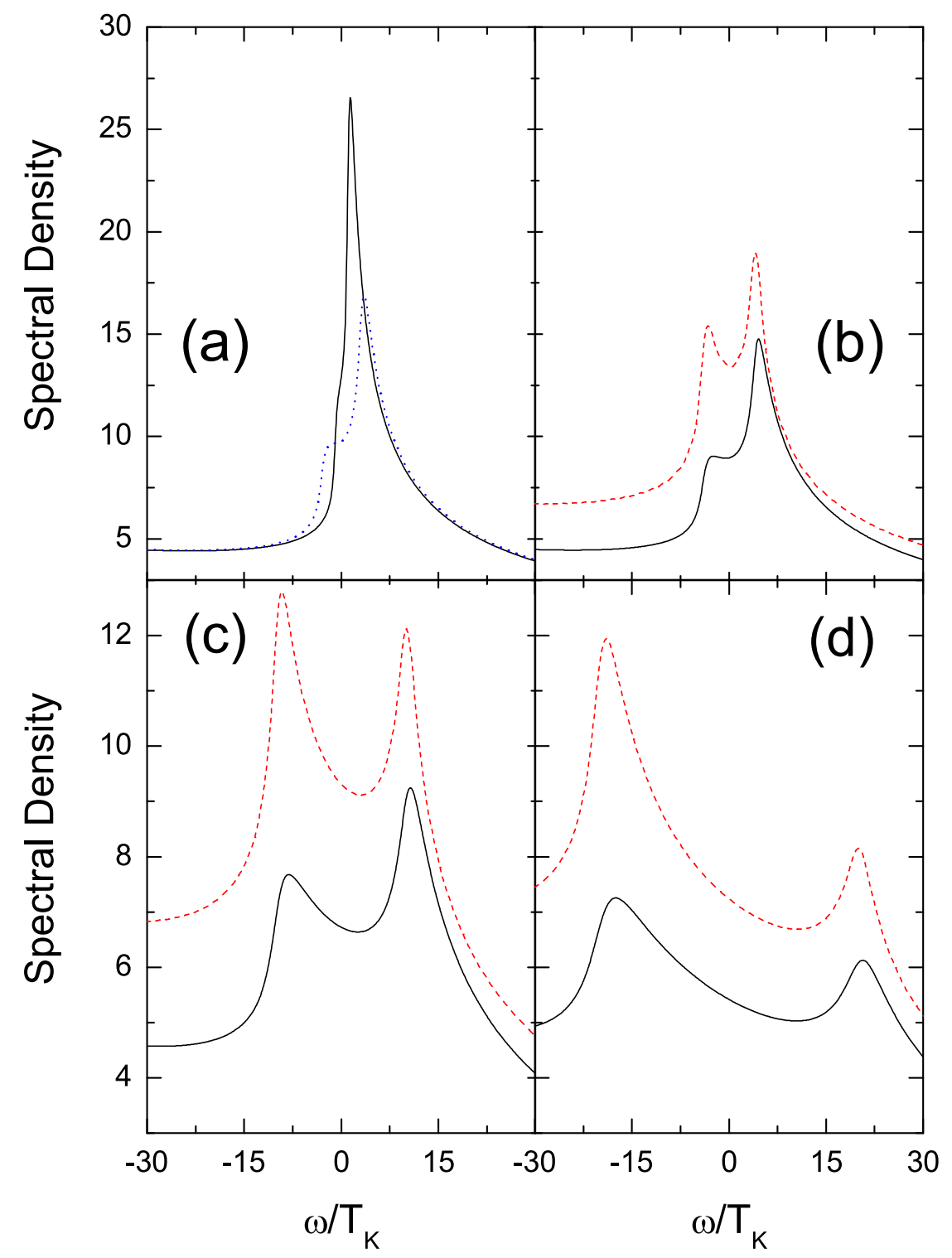

Figure 4: The spectral density in the nonequilibrium regime as a function of $w / T_{K}$, calculated with our EOM method for the same parameters as in Fig.1 is shown for: a) $V=2 T_{K}$ (continuous line) and $V=6 T_{K}$ (dotted line). In Figs.4b, 4c and 4d our results (continuous lines) are compared with the NCA results of Ref.[10] (dashed lines) for: b) $V=8 T_{K}$, c) $V=20 T_{K}$, d) $V=40 T_{K}$. 


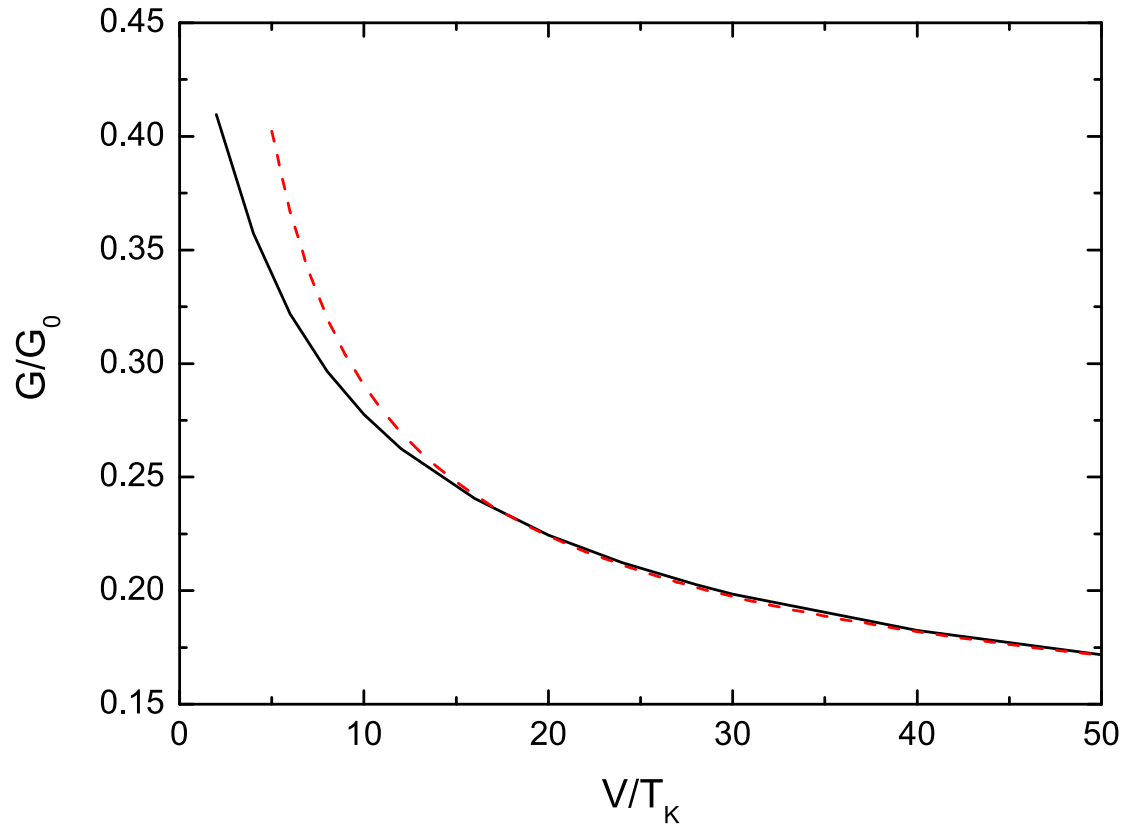

Figure 5: The conductance (in units of the quantum of conductance) calculated for the same parameters as in Figs.1 and 4 (continuous line) is compared with the fitting function of eq.(26) (dashed line). 